\title{
Microscopia eletrônica de varredura da habilidade predatória de agentes do biocontrole de nematóides
}

\author{
Scanning electron microscopy of the predatory ability of agents of the \\ biocontrol of nematodes
}

\author{
Arlete Silveira'; Érika Barbosa Neves Graminha'; Tiago Lot da Silva Nunes; \\ Viviane Aparecida Veronez ${ }^{1}$; Jaime Maia dos Santos²; João Carlos de Oliveira ${ }^{3}$
}

\begin{abstract}
Resumo: Fungos nematófagos têm sido estudados como uma alternativa promissora para o manejo de nematóides de plantas e parasitos gastrintestinais de bovinos. Resultados encorajadores têm sido obtidos com algumas espécies, por vários pesquisadores. A partir de amostras de solo coletadas em diferentes culturas e agroecossistemas provenientes de diversas localidades do Brasil, foram isolados, pelo método de espalhamento de solo, os fungos nematófagos Arthrobotrys javanica, Dactylella leptospora, Monacrosporium eudermatum, M. robustum e Nematoctonus campylosporus, no Centro de Pesquisas em Sanidade Animal (CPPAR) da UNESP/FCAV, Câmpus de Jaboticabal - SP, e a habilidade predatória destes fungos sobre o nematóide de vida livre Panagrellus sp. foi estudada no Laboratório de Microscopia Eletrônica de Varredura. Este trabalho teve como objetivo documentar ao microscópio eletrônico de varredura (MEV) estruturas morfológicas desses fungos tais como: estruturas de captura, tamanho, forma e septação dos conídios, grampos de conexão em $N$. campylosporus, assim como os nematóides de vida livre capturados por estes fungos. Palavras-chave: controle biológico, fungos nematófogos, microscopia eletrônica de varredura.
\end{abstract}

\begin{abstract}
Nematode-trapping fungi have been studied as a promising alternative to control bovine gastrointestinal parasites and plant parasitic nematodes. Some encouraging results have been achieved with some species, by many researchers. Nematophagous fungi such as Arthrobotrys javanica, Dactylella leptospora, Monacrosporium eudermatum, M. robustum e Nematoctonus campylosporus were isolated from soil samples collected from different crops and agronomic environments proceeding from varies places in Brazil, making use of soil spreading methods. These fungi species were isolated at the Center of Animal Parasitology (CPPAR) at UNESP/FCAV, Campus Jaboticabal - SP. The predatory ability of these fungi, on free living nematodes (Panagrellus sp.) was documented at the Scanning Electron Microscopy Lab. The objectives of this work were to document, through Scanning Electron Microscopy (SEM), the morphological structures of these fungi, such as: capturing structures, size, form, septation of the conidia, connection clamp, exclusively on $N$. campylosporus, as well as the free-living nematodes captured by these fungi.
\end{abstract}

Key words: biological control, nematophagous fungi, Scanning Electron Microscopy.

\section{Introdução}

A atividade agrícola tem causado modificações no ambiente, rompendo o balanço natural e favorecendo determinados organismos, indicando que algum fator do balanço biológico não está em equilíbrio (BAKER e COOK, 1974). Disso pode resultar a ocorrência de doenças e pragas de proporções tanto maiores quanto maior for o desequilíbrio. O controle biológico parece ser a restauração do equilíbrio, inibindo a praga ou doença.

Os fungos antagonistas de nematóides podem ser divididos em predadores ou ectoparasitos, endoparasitos, oportunistas (parasitos de ovos, cistos e de fêmeas sedentárias), e aqueles que produzem metabólitos tóxicos aos nematóides (MORGAN-JONES e RODRIGUEZKÁBANA, 1987).

Os fungos predadores ou ectoparasitos formam armadilhas de diferentes tipos (BALAN e GERBER, 1972). São conhecidos seis tipos de armadilhas: hifas adesivas não modificadas; ramificações hifais anastomosadas formando redes adesivas tridimensionais e bidimensionais; ramificações adesivas formadas por uma ou mais células; nódulos adesivos; anéis constritores; e anéis não constritores (GRAY, 1988).

Os principais gêneros de fungos predadores conhecidos são: Arthrobotrys Corda, Dactylaria Saccardo, Dactylella Grove e Monacrosporium Oudemans, os quais parasitam nematóides vermiformes (MANKAU, 1980), podendo algumas espécies parasitar ovos (MAIA, 2000). A ocorrência de fungos predadores e oportunistas tem sido constatada por vários autores a partir de amostras de solo, raízes de plantas e a partir de cistos e/ou massas de ovos de nematóides fitoparasitos (DALLA PRIA et al., 1991; NAVES e CAMPOS, 1991; SANTOS et al., 1991; FERRAZ et al., 1992; MAIA e FERRAZ, 1993;

\footnotetext{
${ }^{1}$ CPPAR-Centro de Pesquisas em Sanidade Animal, FCAV/UNESP, Campus de Jaboticabal.

${ }^{2}$ Departamento de Fitossanidade, FCAV/UNESP, Câmpus de Jaboticabal.

${ }^{3}$ Departamento de Produção Vegetal, FCAV/UNESP, Campus de Jaboticabal.
} 
MAIA et al., 1993; LIMA, 1996; SANTOS, 1996; MIZOBUTS et al., 1997; MIZOBUTSI et al., 1999; RIBEIRO et al., 1999; MAIA, 2000).

Santos (1991) e Dalla Pria (1992) observaram pronunciado efeito antagonista de Monacrosporium ellipsosporum (Grove) Cooke \& Dickinson como um promissor agente para o biocontrole de Meloidogyne incognita (Kofoid \& White) Chitwood, raça 3. Santos (1996) estudou o potencial de 18 isolados de fungos nematófogos, que foram submetidos ao teste, in vitro, de patogenicidade a Heterodera glycines Ichimohe, Pratylenchus brachyurus (Godfrey) Filipvej \& Schuurmans Stekhovem, Meloidogyne arenaria (Meal) Chitwood, M. javanica (Treub) Chitwood e $M$. incognita raça 3. Observou-se que M. ellipsosporum, Arthrobotrys robusta Duddington e três isolados da subdivisão Mastigomycotina foram os mais eficientes quanto ao parasitismo e à predação dos fitonematóides testados, com exceção de $H$. glycines. Sob condições de casa de vegetação em cultivos seqüenciais de feijoeiro, tomateiro e alface, M. ellipsosporum foi o mais eficiente na redução da população desses nematóides.

Santos e Ferraz (2000) avaliaram em casa de vegetação a eficiência de cinco isolados de fungos nematófagos, três isolados da subdivisão Mastigomycotina C9, C22 e CX29, M. ellipsosporum e A. robusta no controle de Meloidogyne arenaria, $M$. incognita raça 3 e $M$. javanica ao longo de três cultivos sucessivos de feijoeiro, tomateiro e alface. O fungo $M$. ellipsosporum foi o mais eficiente no controle dessas três espécies de Meloidogyne, apresentando boa capacidade de sobrevivência no solo, comprovada pela alta taxa de recuperação no final dos três cultivos.

$\mathrm{Na}$ França, foram feitos os primeiros estudos, in vitro, sobre o antagonismo de fungos nematófagos a helmintos. De acordo com Padilha e Gives (1996) e Araújo (1996), a primeira citação de um fungo, capturando nematóide de animais, foi há mais de um século, no final da década de 30. Impulsionados pelos danos causados pelos nematóides e pela baixa eficiência dos métodos de controle da verminose existente naquela época, alguns pesquisadores iniciaram estudos objetivando o controle biológico das formas de vida livre dos nematóides com o uso de fungos nematófagos. Têm sido realizados vários estudos, in vitro, sobre a patogenicidade de fungos nematófagos sobre helmintos de animais domésticos (NANSEN et al., 1988; LARSEN et al., 1991; ARAÚJO et al., 1992; ARAÚJO et al., 1993; WALLER e FAEDO,1993; ARAÚJO et al., 1994; WALLER et al., 1994; GRONVOLD et al., 1995; VIEIRA-BRESSAN et al., 1995; ARAÚJO, 1996; PADILHA e GIVES, 1996; CASTRO et al., 1999; MOTA et al., 1999; NEVES et al., 1999; RODRIGUES et al., 1999; GRAMINHA, 2000; GRAMINHA et al., 2000a; GRAMINHA et al., 2000b).

Estudos utilizando-se da microscopia eletrônica de varredura para documentar o modo de ação destes fungos nematófagos sobre nematóides fitoparasitos (BARRON, 1977; DROWSET e REID, 1977; JANSSON e NORDBRING-HERTZ, 1988; LOPES-LORCA E DUNCAN, 1991; MAIA e SANTOS, 1995; MAIA e SANTOS, 1996;
MAIA E SANTOS, 1997; MAIA e SANTOS, 1999; MAIA, 2000) e sobre helmintos de animais domésticos (PADILHA e GIVES, 1996; GRONVOLD et al., 1996; GRAMINHA, 2000), têm sido realizados por poucos pesquisadores.

Considera-se o controle biológico de nematóides como a redução dos danos causados por estes organismos, por meio da ação de agentes antagonistas. Essa redução de danos poderia ocorrer em função da diminuição na população dos nematóides e/ou pela redução da capacidade de causarem danos. A redução nos danos pode ocorrer naturalmente, pode requerer a manipulação do ambiente ou a introdução em massa de antagonistas. Devido à multiplicidade de fatores envolvidos no complexo doença, o controle satisfatório raramente é alcançado por uma única medida. O controle biológico deve ser, então, considerado como uma das várias medidas complementares em um programa de manejo integrado.

Este trabalho teve como objetivo documentar ao microscópio eletrônico de varredura as principais estruturas morfológicas dos fungos Arthrobotrys javanica (Rifai \& Cooke) Jarowja, Dactylella leptospora Drechsler, Monacrosporium eudermatum (Drechsler) Subram, $M$. robustum McCulloch e Nematoctonus campylosporus Drechsler e nematóides de vida livre (Panagrellus sp.) capturados por estes fungos.

\section{Material e Métodos}

Os ensaios foram conduzidos sob condições de laboratório, do Centro de Pesquisas em Sanidade Animal (CPPAR) e no Laboratório de Microscopia Eletrônica de Varredura da UNESP/FCAV, Câmpus de Jaboticabal (SP). Para a detecção e isolamento dos fungos, foi utilizado o método de espalhamento de solo (BARRON, 1977). Uma alíquota de $2 \mathrm{~g}$ de uma amostra de solo homogeneizada foi colocada no centro de uma placa de Petri contendo ágar-água a $2 \%$. A seguir, foi adicionado $1 \mathrm{~mL}$ de uma suspensão de nematóides de vida livre (NVL) Panagrellus sp. sobre a amostra de solo (SANTOS, 1991). As placas foram mantidas no escuro, à temperatura ambiente $\left(25-28^{\circ} \mathrm{C}\right)$. Inicialmente, as leituras foram diárias, por um período de dez dias, para isolamento de fungos predadores. Depois desse período, as observações foram semanais, estendendo-se por um período de cerca de dois meses. As subculturas dos fungos nematófagos que foram surgindo nas placas, foram preparadas repicando-os para placas de Petri contendo ágar-água a $2 \%$, adicionando-se, a seguir, uma suspensão de NVL. Após o desenvolvimento e esporulação dos fungos, transferiu-se um conídio ou um conjunto (cacho) de conídios para tubos de ensaio contendo o meio de cultura (BDA) inclinado, mantendo esses tubos à temperatura ambiente, até a colonização dos meios pelos fungos.

O preparo das amostras para a documentação ao MEV foi realizado a partir de culturas puras dos fungos nematófagos, as quais foram repicadas para placas de Petri contendo ágar-água a $2 \%$ e incubadas no escuro à temperatura ambiente por 48 horas. A seguir, $1 \mathrm{~mL}$ 
de suspensões concentradas de NVL foi vertido nas placas que foram novamente incubadas como no caso anterior. Quando se observaram 50 ou mais nematóides parasitados e estruturas reprodutivas dos fungos, procedeu-se à fixação em glutaraldeído a $3 \%$, em tampão de fosfato de potássio a 0,05 M e pH 7,2 a 7,4, onde as placas com os fungos e nematóides capturados permaneceram por um período de $72 \mathrm{~h}$. Em seguida, foram lavadas na solução tampão pura e pós-fixadas em tetróxido de ósmio a $2 \%$, no mesmo tampão, por 12 h. Então, foram novamente lavadas, desidratadas em uma série gradual de álcool etílico, secas em secador de ponto crítico, montadas em porta-espécimes, recobertas com uma camada de $36 \mathrm{~nm}$ de ouro paládio e elétron-micrografadas em microscópio eletrônico de varredura JEOL JSM 5410, operando em 15 Kv (MAIA e SANTOS, 1997).

\section{Resultados e Discussão}

Detalhes morfométricos de Arthrobotrys javanica, Dactylella leptospora, Monacrosporium eudermatum, $M$. robustum e Nematoctonus campylosporus e dos nematóides (Panagrellus sp.) capturados foram documentados ao microscópio eletrônico de varredura. As estruturas de captura, tamanho, forma, septação dos conídios, bem como o grampo de conexão, em $N$. campylosporus, foram documentados.

$\mathrm{Na}$ Figura 1, observam-se pormenores do fungo Arthrobotrys javanica; na Figura $1 \mathrm{~A}$, nematóide capturado por rede adesiva tridimensional; na Figura $1 \mathrm{~B}$, detalhe de um nematóide aprisionado pela rede e o rompimento da cutícula deste, pela hifa do fungo; e na Figura 1C, um conidióforo simples, ereto, com células conidiógenas sustentando um conídio bicelular, preso ao esterigma, além de um conídio asseptado, ainda jovem.

A Figura 2 ilustra estruturas de Dactylella leptospora. As estruturas de captura em forma de nódulo e anel não constritor, além de um nematóide aprisionado por essas armadilhas de captura, estão contidos na Figura 2A; em 2B, observa-se um nematóide capturado por nódulos adesivos nas regiões anterior e posterior do corpo; e em $2 \mathrm{C}$ um conidióforo exibindo três esterigmas em seu ápice, além de conídios multisseptados, que se desprenderam do conidióforo, exibindo nódulo em sua extremidade. Estes tipos de armadilhas e conídios são característicos da espécie (BARRON, 1977).

As ilustrações da Figura 3 referem-se a Monacrosporium eudermatum; na Figura $3 \mathrm{~A}$, nematóide com o corpo totalmente parasitado por hifas assimilativas; na Figura 3B, pormenores da hifa tridimensional, a qual difere da rede tridimensional da Figura 1A, por apresentar a extremidade em desenvolvimento, antes de sofrer anastomose, afinando-se a partir da base. Observa-se, ainda, que a rede apresenta formas mais circulares. Na Figura 3C, observa-se conídio ainda jovem, asseptado, preso ao conidióforo.

Monacrosporium robustum está ilustrado na Figura 4; em 4A observa-se um nematóide preso, em três pontos, por uma hifa não diferenciada que apresenta como estruturas de captura os ramos adesivos; na Figura 4B, detalhe de um ramo adesivo, exibindo uma camada fina e transparente que a recobre. Observações semelhantes foram feitas por Barron (1977), ao microscópio eletrônico de varredura, para as estruturas de captura de Arthrobotrys oligospora. Na Figura 4C, está ilustrado um conídio, apresentando quatro septos, preso ao conidióforo. Este fungo foi detectado pela primeira vez, no Brasil, em solos provenientes de plantações de seringais, em Itiquira - MS. (MAIA et al., 2001). Em testes, in vitro, realizados por esses mesmos autores, o fungo $M$. robustum mostrou-se promissor contra nematóides fitoparasitos, tais como: Heterodera glycines Ichinihe (nematóide de cisto da soja), Meloidogyne incognita (Kofoid \& White) Chitwood e M. javanica (Treub) Chitwood (nematóides das galhas). Severas perdas na agricultura em todo o mundo são atribuídas à ação dos nematóides das galhas (SANTOS e FERRAZ, 1991) e ao nematóide de cisto da soja (SASSER, 1989; ESTRATÉGIAS..., 1998).

$\mathrm{Na}$ Figura 5A, B e C, estão representadas estruturas de Nematoctonus campylosporus. Na Figura 5A, observa-se um nematóide preso na região anterior do corpo, por um nódulo adesivo. Não há relatos na literatura de que a hifa deste fungo seja também aderente. Porém, nesta figura, pede-se observar o contato direto da hifa no corpo do nematóide. Em 5B, detalhe da hifa com septo tipo grampo de conexão, característico dos fungos da subdivisão Basidiomycotina (MUCHOVEJ e MUCHOVEJ, 1989), e na Figura 5C, a sua estrutura reprodutiva. Os conídios deste fungo são formados diretamente ao longo da hifa ou em pequenos conidióforos que emergem do corpo dos nematóides parasitados (BARRON, 1977).

Os tipos de armadilhas e estruturas reprodutivas documentados no presente estudo estão de acordo com os observados pelos pesquisadores que os descreveram. Arthrobotrys javanica foi descrita por Rifai e Cooke (1966) como Candelabrellajavanica; Dactylella leptospora, Monacrosporium eudermatum e Nematoctonus campylosporus foram descritas por Drechsler (1937, 1950 ,1954). Porém, para M. robustum, Mcculloch, (1977), relatou que esta espécie apresentava, como órgão de captura, nódulos adesivos. Com as observações feitas no presente estudo, com auxílio da MEV, constatou-se que os órgãos de captura são ramos adesivos, formados sempre por uma célula. Esta célula nunca se desprende da hifa como geralmente ocorre com os fungos formadores de nódulos adesivos, diferenciandoos, também, de M. cionopagum (Drechsler) Mekhtieva onde os órgãos de captura são ramos adesivos formados por uma ou mais células.

A microscopia eletrônica de varredura é uma das ferramentas mais eficazes para auxiliar o entendimento do modo de ação dos fungos nematófagos e revelar pormenores de suas estruturas reprodutivas e de captura. 

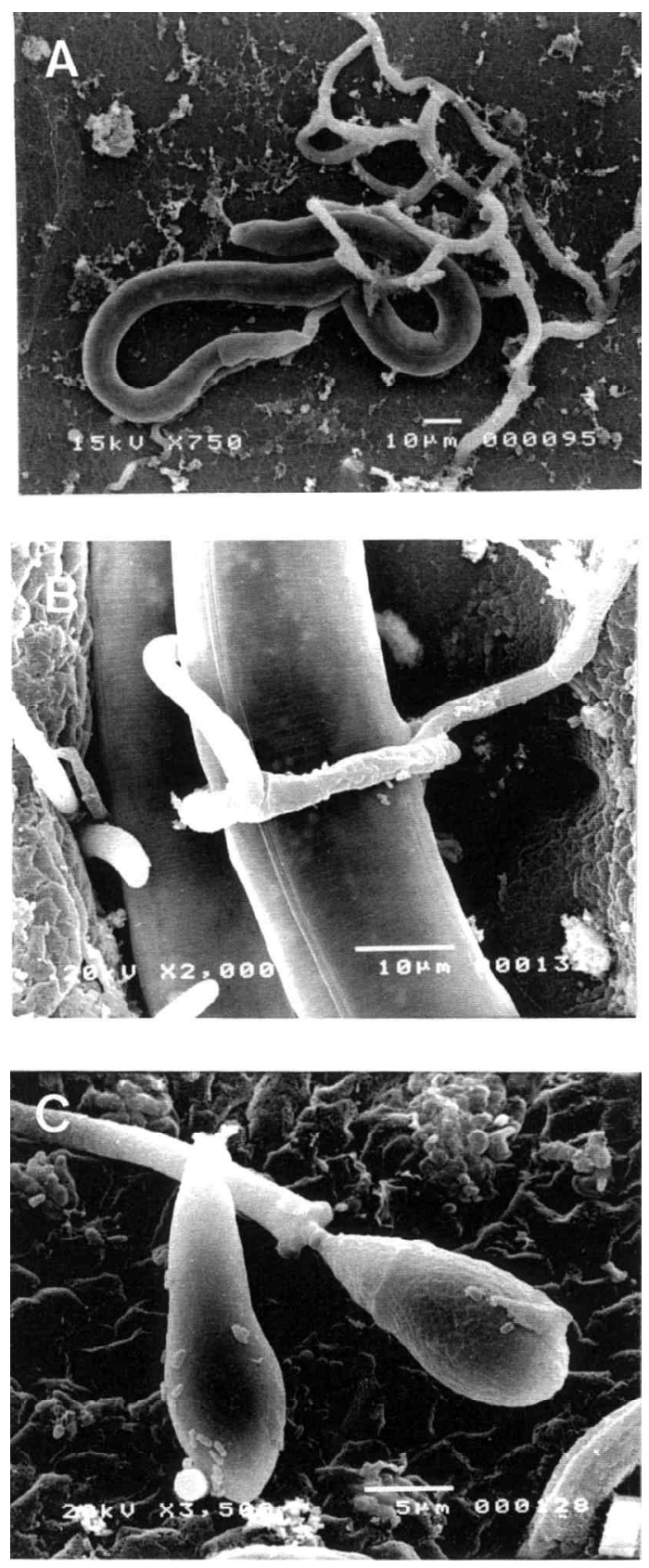

Figura 1 - Elétron-micrografia de varredura de Arthrobotrys javanica. A) Nematóide capturado por redes tridimen-sionais. B) Detalhe do nematóide aprisionado pela rede adesiva. C) Um conídio ainda jovem, asseptado, e um conidióforo sustentando um conídio bicelular preso ao esterigma.
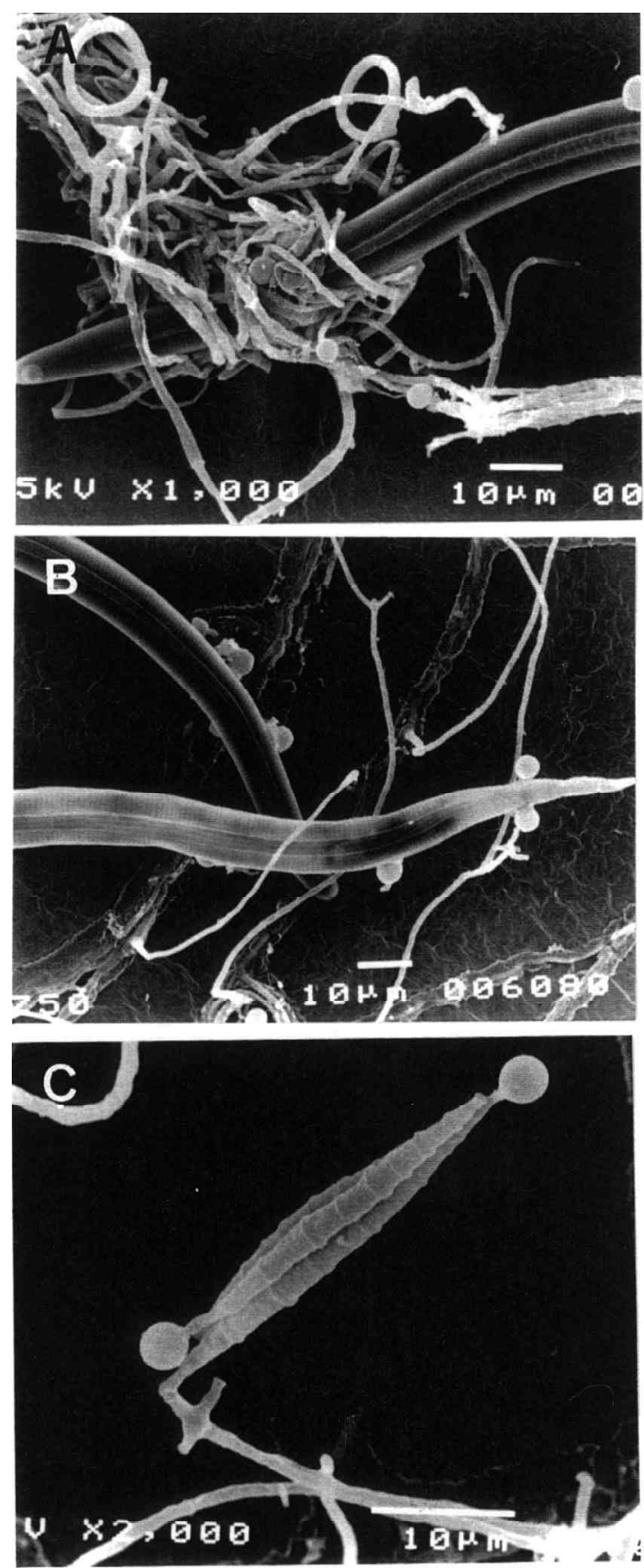

Figura 2 - Elétron-micrografia de varredura de Dactylella leptospora. A) Estrutura de captura em forma de nódu-lo e anel não constritor presos à hifa vegetativa, além de um nematóide aprisionado por estas armadilhas de captura. B) Nematóide capturado por nódulos adesivos. C) Conidióforo com três esterigmas em seu ápice, e conídios exibindo nódulo em sua 

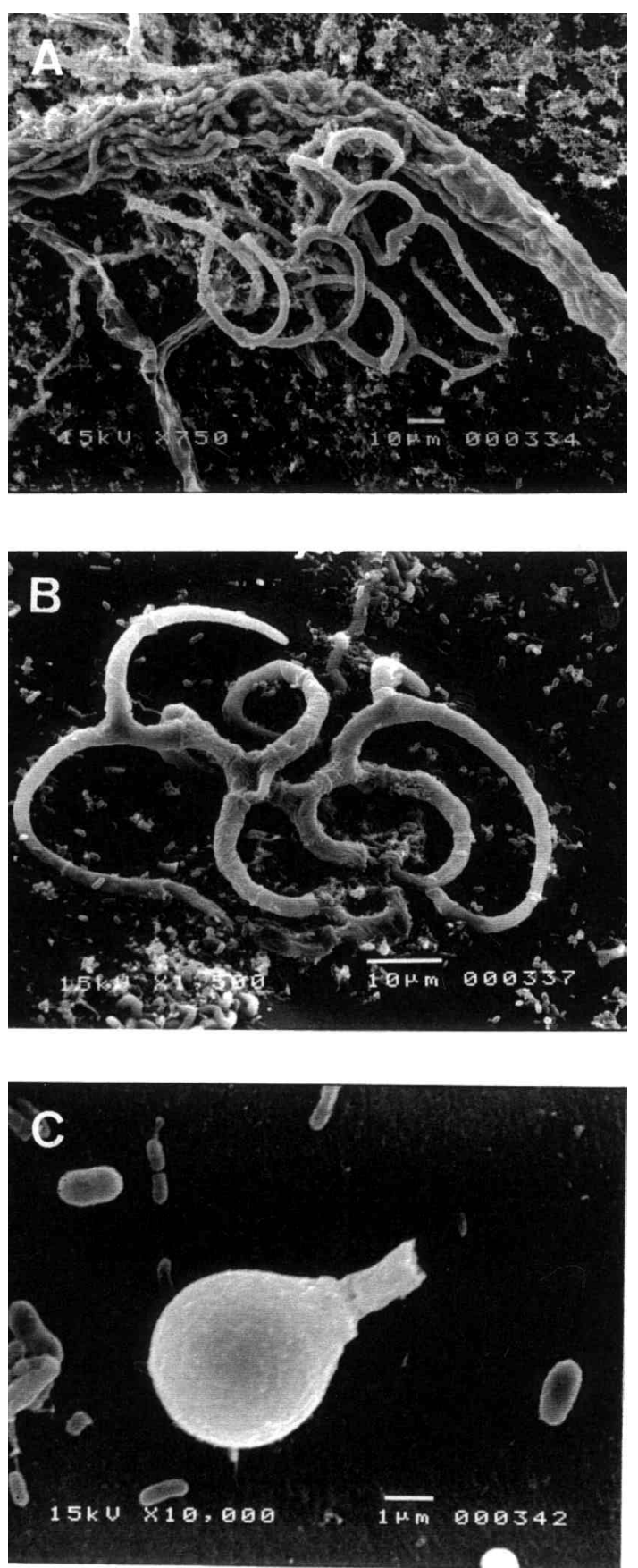

Figura 3 - Elétron-micrografia de varredura de Monacrosporium eudermatum. A) Nematóide com o corpo totalmente parasitado por hifas. B) Detalhes da hifa tridimensional. C) Conídio ainda jovem, asseptado, preso em parte do conidióforo.
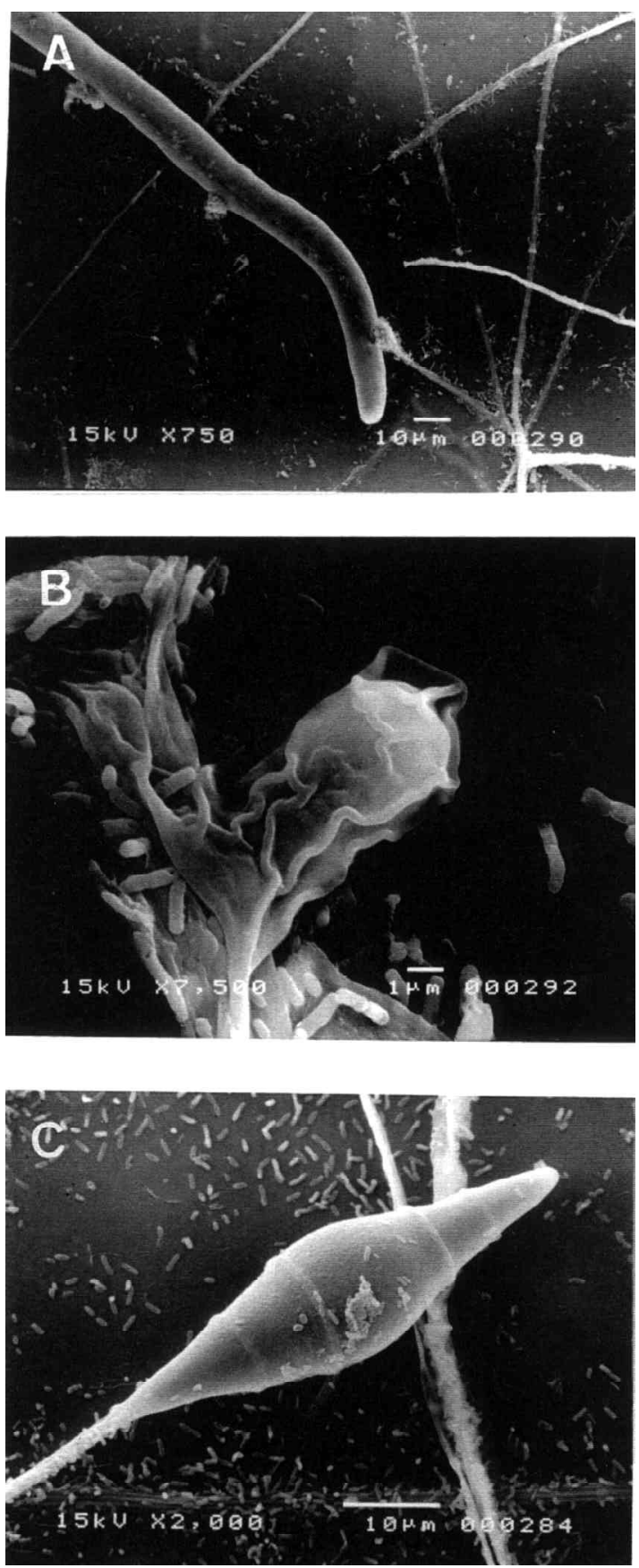

Figura 4 - Elétron-micrografia de varredura de Monacrosporium robustum. A) Nematóide preso, em três pontos, por hifa de tipo ramo adesivo. B) Detalhe de um ramo adesivo e a parte adesiva da hifa. C) Conídio, apresentando quatro septos, preso ao conidióforo. 

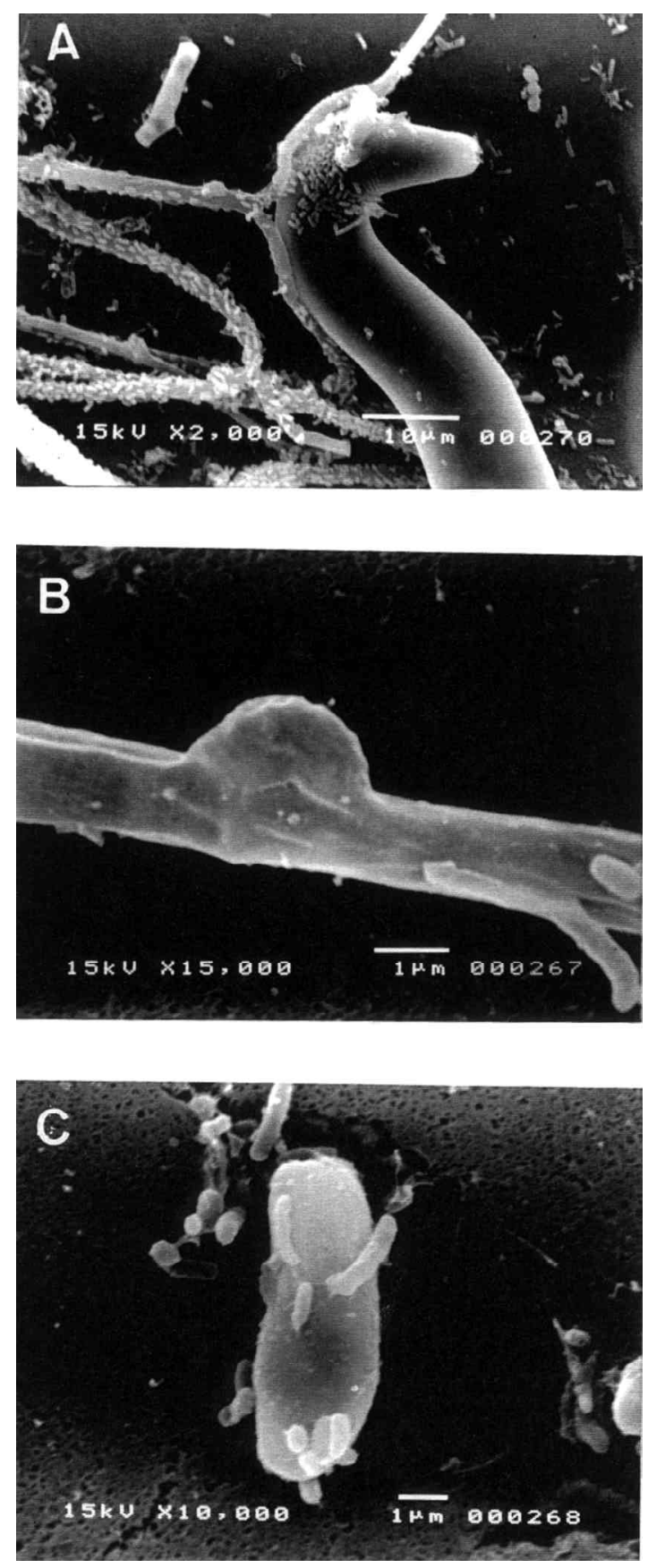

Figura 5 - Elétron-micrografia de varredura de Nematoctonus campylosporus. A) Nematóide preso por um nódulo adesivo. B) Hifa com grampo de conexão. C) Estrutura reprodutiva do fungo.

\section{Referências Bibliográficas}

ARAUJO, J.V. de. Interação entre larvas infectantes de Cooperia punctata e fungos predadores do gênero Arthrobotrys, caracterização de isolados de Arthrobotrys e seu uso no controle biológico de nematódeos parasitos gastrintestinais de bovinos. 1996. 110f . Tese (Doutorado em Ciências) - Instituto de Ciências Biológicas, Universidade Federal de Minas Gerais. Belo Horizonte, 1996.

ARAUJO, J.V. de. et al.. Antagonistic effect of predacious Arthrobotrys fungi in infective Haemonchus placei larva. J. of Helminthol., London, v.67, p.136-138, 1993.

ARAUJO, J.V. et al.. Biological control in vitro of infective Haemonchus placei larva by predacious fungi Arthrobotrys musiformis. Arq. Bras. Med. Vet. Zootec., Belo Horizonte, v.46, p.194-204, 1994.

ARAUJO, J.V. de. et al.. Controle de larvas infectantes de Haemonchus placei por fungus predadores da espécie Monacrosporium ellypsosporum em condições de laboratório. Arq. Bras. Med. Vet. Zootec., Belo Horizonte, v.44, p.521-526, 1992.

BAKER, K. F.; COOK, R. J. Biological control of plant pathogens. São Francisco: W. H. Freeman 1974. 433p.

BALAN, J.; GERBER, N. Attraction and killing of nematode Panagrellus redivivus by the predaceus fungus Arthrobotrys dactyloides. Nematologica, Leiden, v.18, n.1, p.163-173. 1972.

BARRON, G.L. The mematode-destroying fungi. Guelph: Canadian Biological Publications, 1977. 140 p.

CASTRO, A. A. et al.. de. Avaliação do fungo Monacrosporium thaumasium (isolado - NF34a) sobre larvas pré-parasíticas de Cyatostominae (Nematoda-Strongylidae) em coproculturas. In: SEMINÁRIO

BRASILEIRO DE PARASITOLOGIA VETERINÁRIA, 11, 1999, Salvador. Anais... Salvador: CBPV, 1999. p.165.

DALLA PRIA, M. Controle biológico de Meloidogyne incognita raça 3, pelos fungos Verticillium chlamidosporium e espécies de Monacrosporium isolados ou combinados. 1992. 101f. Dissertação (Mestrado em Fitopatologia) Departamento de Fitopatologia, Universidade Federal de Viçosa, Viçosa, 1992.

DALLA PRIA, M.; FERRAZ, S.; MUCHOVEJ, J.J. Isolamento e identificação de fungos nematófagos de amostras de solo de diversas regiões do Brasil. Nematol. Bras., Campinas, v.15, n.1, p.170-178. 1991.

DOWSETT, J.A.; REID, J. Observations on the trapping of nematodes by Dactylaria scaphoides using optical, transmission and scanning-electron-microscopic techniques. Mycology, New York, v.71, p.379-391, 1977.

DRECHSLER, C. A nematode-capturing fungus with clampconnections and curved conidia. J. of Washington Acad. Science, Washington, v.44, p.82-85, 1954.

DRECHSLER, C. Several species of Dactylella and Dactylaria that capture free-living nematodes. Mycology, New York, v.42, p.1-79,1950.

DRECHSLER, C. Some hyphomycetes that prey on freeliving terricolous nematodes. Mycology, New York, v.29, p.447-553, 1937.

ESTRATÉGIAS de controle das doenças da soja. Corrêio Agrícola(São Paulo), São Paulo, v.2. p. 8-13, 1998.

FERRAZ, S. et al.. dos. Detecção, isolamento, identificação e avaliação in vitro da capacidade predatória de fungos nematófagos de solos brasileiros. Nematol. Bras., Campinas, v.16, n.1, p.85-86, 1992.

GRAMINHA, E.B.N. Avaliação in vitro da patogenici-dade de fungos predadores de nematóide parasitos de animais domésticos. 2000. 94f. Dissertação (Mestrado em Microbiologia) - Departamento de Microbiologia, Universidade Estadual Paulista, Jaboticabal, 2000.

GRAMINHA, E.B.N. et al.. Avaliação in vitro da patogenicidade de fungos predadores de nematóide parasitos de animais domésticos. In: FÓRUM EM MICROBIOLOGIA, 3, 2000, Jaboticabal. Resumos... Jaboticabal: FUNEP, 2000a. p.11. 
GRAMINHA, E.B.N. et al.. Controle biológico de helmintos: avaliação predatória de fungos nematófagos em larvas de nematóides de ovinos. In: FÓRUM EM MICROBIOLOGIA, 3, 2000, Jaboticabal. Resumos... Jaboticabal: FUNEP, 2000b. p.15.

GRAY, N.F. Fungi attacking vermiform nematode. In: POINAR JUNIOR, G.O.; JANSSON, N.B. Disease of nematodes. Boca Raton: CRC Press, 1988. p.3-38.

GRONVOLD, J. et al.. Laboratory experiments to evaluate the ability of Artrhobotrys oligospora to destroy larvae of Cooperia species, and to investigate the effect of physical factors on the growth of the fungus. J. of Helminthol., London, v.59, p.119-125,1995.

GRONVOLD, J. et al.. Induction of traps by Ostertagia ostertagi larva, chlamydospore prodiction and growth rate in the nematode-trapping fungus Duddingtonia flagrans. J. of Helminthol., London, v.70, p.291-297,1996.

JANSSON, H.B.; NORDBRING-HERTZ, B. Infection events in the fungus-nematode system. In: PAINAR Fr., G.O.; JANSSON, H.G. Disease of nematodes. Boca Raton: CRC Press., 1988, v.2. p.59-72.

LARSEN, M. et al.. In vitro stress selection of nematophagous fungi for biocontrol of parasitic nematodes in ruminants. J. Helminthol., London, v.65, n.3, p.193-200, 1991.

LIMA, R.D. Caracterização de Isolados e Avaliação da Patogenicidade de Arthrobotrys spp. a Fitonema-tóides. 1996. 88f. Tese (Doutorado em Fitopotologia)Departamento de Fitopotologia, Universidade Federal de Viçosa, Viçosa, 1996.

LOPEZ-LLORCA, L.V.; DUNCAN, G.H. Effect of fungal parasitic cyst nematode (Heterodera avenae Woll.) from naturaly infest soil - a scanning electron microscopy study. Can. J. Microbiol., Ottawa, v.37, p.218-225, 1991.

MAIA, A.S. Isolamento, identificação e potencialidade de fungos como agentes de biocontrole de Meloidogyne spp. e Heterodera glycines. 2000. 117f. Tese (Douto-rado em Agronomia) - Departamento de Produção Vegetal, Universidade Estadual Paulista de Jaboticabal, Jaboticabal, 2000.

MAIA, A.S.; FERRAZ, S. Detecção e isolamento de fungos nematófagos de solos brasileiros. In: CONGRESSO BRASILEIRO DE NEMATOLOGIA, 17, 1993, Jaboticabal, Resumos... Jaboticabal: Sociedade Brasileira de Nematologia, 1993. p.68.

MAIA, A.S.; FERRAZ, S.; DALLA PRIA, M. Detecção, isolamento e identificação de Monacrosporium spp. In: CONGRESSO BRASILEIRO DE NEMATOLOGIA, 17; 1993, Jaboticabal, Resumos... Jaboticabal: Sociedade Brasileira de Nematologia, 1993. p.69.

MAIA, A.S.; SANTOS, J.M. dos. Microscopia eletrônica de varredura das estruturas de captura de alguns fungos nematófagos. In: INTERAMERICAM CONFERENCE ON ELECTRON MICROSCOPY, 3, 1995, Caxambu. Anais... Caxambu: [s.n.], 1995. p.304.

MAIA, A.S.; SANTOS, J.M. dos. Novos dados obtidos ao microscópio eletrônico de varredura sobre fungos nematófagos. In: CONGRESSO BRASILEIRO DE FITOPATOLOGIA, 29, 1996, Campo Grande. Resumos... Campo Grande: Sociedade Brasileira de Fitopatologia, 1996. p.370.

MAIA, A.S., SANTOS, J.M. dos. A SEM technique for preparing biological control agents of nematodes in action. Acta Microscopica, Caracas, v.7, Suppl. B, 1997. p.550.
MAIA, A.S.; SANTOS, J.M. dos. SEM study of nematophagous fungi in action. Acta Microscopica, Caracas, v.8, Suppl. C, 1999. p.625-617.

MAIA, A.S.; SANTOS, J.M. dos.; DI MAURO, A.O. Estudo in vitro da habilidade predatória de Monacrosporium robustum sobre Heterodera glycines. Fitopatol. Bras., Brasília, v.26, n.4, 2001.

MANKAU, R. Biocontrol: fungi as nematode control agents. Journal of Nematology, Jay, v.12, p.244-252, 1980.

McCULLOCH, J.S. New species of nematophagous fungi from Queensland. Transactions of the Britsh Mycological Society, Cambridge, v.68, p.173-179, 1977.

MIZOBUTSI, E.H. et al.. Isolamento de fungos de ovos de Heterodera glycines coletados em diferentes regiões produtoras de soja do Brasil. Nematol. Bras., Campinas, v.23, n.2, p.69-75, 1999.

MIZOBUTSI, E.H. et al.. Detecção e isolamento de fungos parasitos de ovos de Heterodera glycines em diferentes regiões produtoras de soja. In: CONGRESSO BRASILEIRO DE NEMATOLOGIA, 20, 1997, Gramado. Resumos... Gramado: Sociedade Brasileira de Nematologia, 1997. p.69.

MORGAN-JONES, G.; RODRIGUEZ-KÁBANA, R. Fungal biocontrol for the management of nematode. In: VEECH, J. A.; DICKINSON, D. W. Vistas on nematology. Maryland: Society of Nematologists, 1987. p.49-88.

MOTA, M. et al.. Comparação da atividade predatória de fungos nematófagos das espécies de Arthrobotrys conoides e Monacrosporium thaumasium sobre larvas infectantes de Haemonchus contortus. In: SEMINÁRIO BRASILEIRO DE PARASITOLOGIA VETERINÁRIA, 11, 1999, Salvador. Anais... Salvador: CBPV, 1999. p.156.

MUCHOVEJ, J.J.; MUCHOVEJ, R.M.C. Noções básicas de Micologia. Viçosa: Folha de Viçosa, 1989. 155p.

NANSEN, P. et al.. Interactions between the predacious fungus Arthrobotrys oligospora and third-stage larvae of a series of animal-parasitic nematodes. Veter. Parsitol., Amsterdan, v.26, 329-337, 1988.

NAVES, R.L.; CAMPOS, V.P. Ocorrência de fungos predadores de nematóides no Sul de Minas Gerais e estudos da capacidade predatória e crescimento in vitro de alguns de seus isolados. Nematol. Bras., Campinas, v.15, n.1, p.152-162. 1991.

NEVES, E.B. et al.. Avaliação da patogenicidade de fungos nematófagos sobre ovos de Ascaridia galli. In: SEMINÁRIO BRASILEIRO DE PATOLOGIA VETERINÁRIA, 11; SEMINÁRIO DE PARASITOLOGIA VETERINÁRIA DOS PAÍSES DO MERCOSUL, 2, SIMPÓSIO DE CONTROLE INTEGRADO DE PARASITOS DE BOVINOS, 1, 1999, Salvador. Anais... Salvador: Colégio Brasileiro de Parasitologia Veterinária, 1999. p.177.

PADILHA, T.; GIVES, P.M. de. Controle microbiano das formas de vida livre dos nematódeos trichostrongilídeos: uma alternativa para higienização das pastagens. In: PADILHA, T. Controle dos nematódeos gastrointestinais em ruminantes. Coronel Fabriciano: EMBRAPA-CNPGL,1996. p.215-235.

RIBEIRO, R.C.F.; FERRAZ, S.; MIZOBUTSI, E.H.; MENDEZ, M. Levantamento de espécies de Monacrosporium predadores de nematóides em diversas regiões brasileiras. Nematologia Brasileira, Campinas, v.23, n.2, p.40-47, 1999. 
RIFAI, M.A.; COOKE, R.C. Studies on some Didymosporous genera of nematode-trapping hyphomycetes. Trans. Br. Mycol. Soc. Cambridge, v.49, n.1, p.147-168, 1966.

RODRIGUES, M.L.A. et al.. Avaliação da capacidade predatória de Monacrosporium thaumasium (NF34a) sobre larvas infectantes de Cyathostominae (observações preliminares). In: SEMINÁRIO BRASILEIRO DE PARASITOLOGIA VETERINÁRIA, 11, 1999, Salvador. Anais... Salvador, CBPV, 1999. p.164.

SANTOS, M. A. Detecção, identificação e avaliação do potencial antagonista de fungos nematófagos em solos do Brasil. 1991. 97f. Dissertação (Mestrado em Fitopatologia) - Departamento de Fitopatologia, Universidade Federal de Viçosa, Viçosa, 1991.

SANTOS, M. A. Estudo de alguns fungos endoparasitos e predadores no controle de fitonematóides. 1996. 166f. Tese (Doutorado em Fitopatologia). Departamento de Fitopatologia, Universidade Federal de Viçosa, Viçosa, 1996.

SANTOS, M.A.; FERRAZ, S. Eficiência de cinco isolados de fungos nematófagos no controle de Meloidogyne spp. ao longo de três cultivos sucessivos. Nematologia Brasileira, Campinas, v.24, n.2, p.193-201, 2000.

SANTOS, J.M. dos; FERRAZ, S. Microscopia eletrônica de varredura de esporângios de Pasteuria penetrans aderidos à cutícula de larvas de Meloidogyne javanica. In: COLÓQUIO DA SOCIEDADE DE MICROSCOPIA ELETRÔNICA, 12, 1991, Caxambu. Resumos... Rio de Janeiro: SBME, 1991. p.301-302.

SANTOS, M.A.; FERRAZ, S.; MICHOVEJ, J.J. Detection and ecology of nematophagous fungi from Brazilian soils. Nematologia Brasileira, Campinas, v.15, n.1, p.121-134. 1991.

SASSER, J.N. Plant-parasitic nematodes the farmer's hidden enemy. Raleigh: North Carolina State University, 1989. 115p.

TUNLID, A.; JANSON, S. Protease and ther involvement in the infection and immobilization of nematodes by the nematophagous fungus Arthrobotrys oligospora. Appl. and Environ. Microbiol., Washington, v.57, n.10, p.2868-2872, 1991.

TUNLID, A.; ROSEN, S.; NORDBRING-HERTZ, B. Molecular mechanisms of adhesion in the nematophagous fungus Arthrobotrys oligospora. J. Mycol. Méd. Paris, v.2, n.1, p. 36-42, 1992.

VIEIRA-BRESSAN, M.C.R. et al.. Pathophysiological observation on calves concurrently infected with Cooperia punctata and Haemonchus placei. Arg. Bras. Med. Vet. Zoot., Belo Horizonte, v.47, p.53-64, 1995.

WALLER, P.J.; FAEDO, M. The potential of nematophagous fungi to control the free-living stages of nematode parasites of sheep: screening studies. Vet. Parasitol.,Amsterdam, v. 49, 285-297, 1993.

WALLER, P.J. et al.. The potencial of nematophagous fungi to the potential of nematophagous fungi to control the freeliving stages of nematode parasites of sheep: in vitro studies. Vet. Parasitol., Amsterdam, v.51, 289-99, 1994. 